Document downloaded from:

http://hdl.handle.net/10251/121371

This paper must be cited as:

Bañón, D.; Socuellamos, JM.; Mata-Sanz, R.; Mercadé-Morales, L.; Gimeno Martínez, B.; Boria Esbert, VE.; Raboso García-Baquero, D.... (2018). Study of the Secondary Electron Yield in Dielectrics Using Equivalent Circuital Models. IEEE Transactions on Plasma Science. 46(4):859-867. https://doi.org/10.1109/TPS.2018.2809602

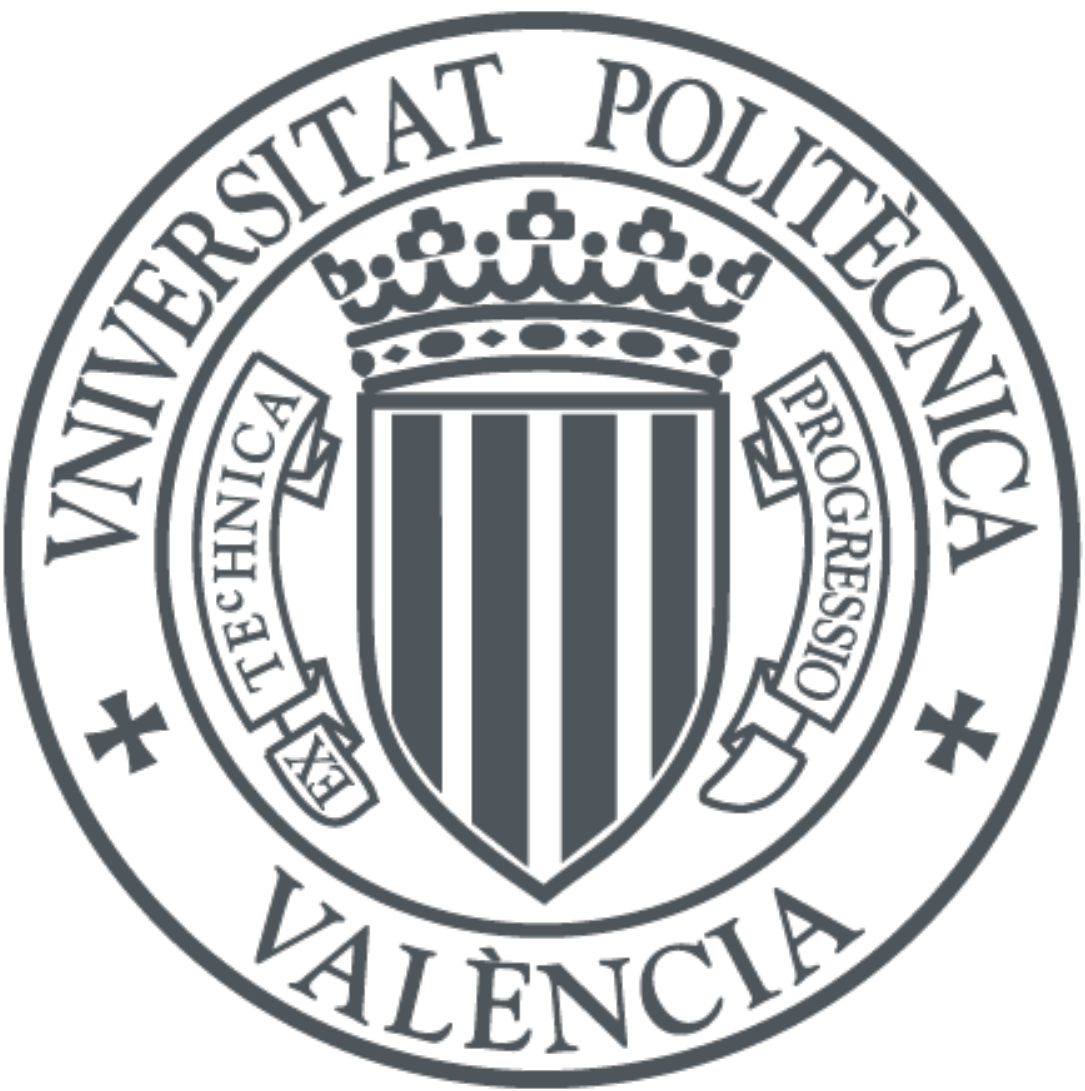

The final publication is available at

http://doi.org/10.1109/TPS.2018.2809602

Copyright Institute of Electrical and Electronics Engineers

Additional Information 


\title{
Study of the Secondary Electron Yield in Dielectrics Using Equivalent Circuital Models
}

\author{
David Bañón-Caballero, Juan M. Socuéllamos, Rafael Mata, Laura Mercadé, Benito Gimeno ${ }^{\circledR}$, Member, IEEE, \\ Vicente E. Boria, Fellow, IEEE, David Raboso, Vladimir E. Semenov, \\ Elena I. Rakova, Juan F. Sánchez-Royo, and Alfredo Segura
}

\begin{abstract}
Secondary electron emission has an important role on the triggering of the multipactor effect; therefore, its study and characterization are essential in radio-frequency waveguide applications. In this paper, we propose a theoretical model, based on equivalent circuit models, to properly understand charging and discharging processes that occur in dielectric samples under electron irradiation for secondary electron emission characterization. Experimental results obtained for $\mathrm{Pt}, \mathrm{Si}, \mathrm{GaS}$, and Teflon samples are presented to verify the accuracy of the proposed model. Good agreement between theory and experiments has been found.
\end{abstract}

Index Terms-Multipactor effect, radio frequency, secondary electron emission (SEE), secondary electron yield.

\section{INTRODUCTION}

$\mathbf{I}^{\mathrm{N}}$ $\mathrm{N}$ RADIO frequency (RF) applications, such as satellite communications or particle accelerators, the multipactor effect [1] may appear limiting the power of the electromagnetic waves [2]-[7]. Under certain conditions, electrons produced by secondary electron emission (SEE) and also electrons from external sources may couple with the alternating electric field generating an electron avalanche. This produces an electron cloud which may lead to disturbances on the measurements or even the destruction of the devices in the worst case scenario.

To characterize the multipactor effect, it is needed to know the ratio between the outgoing and incoming electrons on a material surface. This ratio is called secondary electron

Manuscript received June 5, 2017; revised January 16, 2018; accepted February 18, 2018. Date of publication March 8, 2018; date of current version April 10, 2018. The review of this paper was arranged by Senior Editor A. I. Smolyakov. (Corresponding author: Benito Gimeno.)

D. Bañón-Caballero is with the European Organization for Nuclear Research (CERN), 1211 Geneva, Switzerland (e-mail: david.banon.caballero@cern.ch).

J. M. Socuéllamos is with the Engineering Department, Lancaster University, Lancaster LA1 4YW, U.K. (e-mail: j.socuellamos@lancaster.ac.uk).

R. Mata is with the Val Space Consortium, 46100 Valencia, Spain (e-mail: rafael.mata@uv.es).

L. Mercadé, B. Gimeno, J. F. Sánchez-Royo, and A. Segura are with the Department of Applied Physics and Electromagnetism, Instituto de Ciencia de Materiales, Universidad de Valencia, Valencia 46100, Spain (e-mail: mermolau@alumni.uv.es; benito.gimeno@uv.es; juan.f.sanchez@uv.es; alfredo.segura@uv.es).

V. E. Boria is with the Department of Communications, Instituto de Telecomunicaciones y Aplicaciones Multimedia, Universidad Politécnica de Valencia, 46022 Valencia, Spain (e-mail: vboria@dcom.upv.es).

D. Raboso is with the European Space Agency, European Space Research and Technology Center, 2200 AG Noordwijk, The Netherlands (e-mail: david.raboso@esa.int)

V. E. Semenov and E. I. Rakova are with the Institute of Applied Physics, Russian Academy of Science, Nizhny Novgorod 603950, Russia (e-mail: sss@appl.sci-nnov.ru)

Color versions of one or more of the figures in this paper are available online at http://ieeexplore.ieee.org.

Digital Object Identifier 10.1109/TPS.2018.2809602 yield (SEY) or $\sigma$, and if it is higher than 1, the multipactor effect can be triggered ON. At this point, it is important to stress that we do not make distinctions on the character of the outgoing electrons, since it is irrelevant for the purpose of this paper; however, its classification can be consulted in [8].

There have been many studies of SEE curves in the technical literature. Beginning with the first articles [9]-[11], where the first experiments to measure SEE were described, we can find more recent works that address this problem which is especially complex in the case of dielectrics. Every material presents a characteristic SEY which also depends on the primary electron energy $E_{p}$; in this line, it is common to represent SEY versus $E_{p}$ curves on experimentl results [12] and theoretical simulations [13]. A qualitative scheme of the parts of an SEY curve can be found in [14].

When manufacturing RF spacecraft devices, metals and/or dielectrics are widely used, depending on each particular application. Because of that, it is important to study the $\sigma$ coefficient for both kinds of materials. According to the works found in the technical literature, one can notice that the measurement process is well known for metals, and $\sigma$ can be measured with pretty good precision. On the other hand, dielectric materials present charging effects that disturb the SEE measurements. Due to their electrical properties, the technique is not as clearly defined as for metals, and it should be improved.

This paper is focused on the study of the charging processes that affect dielectric samples. Pt, $\mathrm{Si}, \mathrm{GaS}$, and Teflon samples have been analyzed under direct current (dc) electron incidence and also with pulsed irradiation, in order to observe differences in terms of the SEE behavior. To understand the results obtained, the setup and the sample have been modeled with an electrical equivalent circuit. The solutions found for the proposed circuit fit also the measured experimental curves pretty well, taking some physical considerations into account.

This paper is organized as follows. In Section II, the experimental setup and the two different techniques used throughout the experiments are defined. Section III deals with the equivalent circuit model proposed in this paper. In Section IV, experimental results and simulations of the secondary current versus time or versus $E_{p}$ are shown. Finally, in Section V, a summary of the main conclusions of this paper is presented.

\section{SEY Measurements And Experimental Setup}

\section{A. SEY Measurements Analysis}

One of the techniques most commonly used to measure the SEY coefficient consists of using samples as thin as 

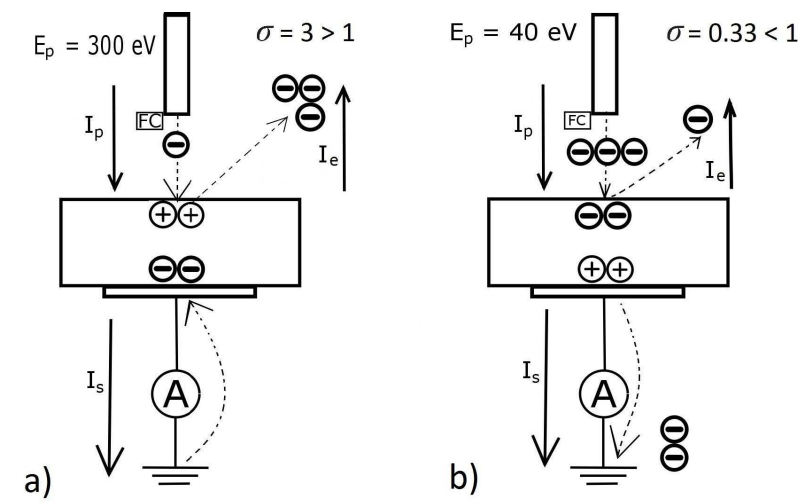

Fig. 1. Schematic of the current balance for a typical SEY measurement with the sample connected to ground. Thick arrows mean normal currents, whereas the dashed ones are the electrons trajectories. Clear differences are observed between (a) $\sigma>1$ process and (b) opposite one.

possible, taking into account that the primary electron incidence together with the SEE produces charging variations on the surface of the sample. Then, in order to reach the electrostatic equilibrium, a compensatory current from ground appears. This current is measurable and allows to evaluate the SEY coefficient. An example of the complete process is represented in Fig. 1, where $I_{p}, I_{e}$, and $I_{s}$ represent the primary, the secondary, and the compensatory currents, respectively. Then, applying the current law to the node, we have the relationship $I_{p}=I_{e}+I_{s}$. Now, we clarify the current signs: $I_{p}$ and $I_{e}$ are always negative, whereas $I_{s}$ changes its sign depending on the situation. In Fig. 1(a), the SEY is higher than 1 and $I_{S}$ is a positive current. On the other hand, in Fig. 1(b), the SEY is lower than 1 and $I_{s}$ is negative.

According to Fig. 1, one can relate the measured currents and the SEY coefficient as follows:

$$
\sigma=\frac{I_{e}}{I_{p}}=1-\frac{I_{s}}{I_{p}}
$$

\section{B. Experimental Setup and Processes}

To perform our experiments, we made use of an X-Ray/Ultraviolet Photoelectrons Spectroscopy system within a clean room class 100000 (ISO8), located at the European High Power Space Materials Laboratory, European Space Agency,Val Space Consortium [15]. This apparatus allows to reach $10^{-10}$ mbar in the analysis chamber and incorporates a Kymball Physics ELG2 electron gun.

When $I_{p}$ is measured, using a Faraday cup, a positive bias voltage of $+57 \mathrm{~V}$ is chosen to produce a potential well ensuring that all the primary electrons are taken into account. On the other hand, for measuring $I_{s}$, a negative bias of $-28 \mathrm{~V}$ is used to prevent that the secondary electrons return to the sample once emitted. This procedure ensures measurements quality, but the primary energy is shifted because of the bias voltage; this correction must be performed when processing the experimental data.

After doing these general clarifications, the explanation follows with the distinction of the two different operation modes that are used in this paper.
1) Continuous Mode: On this first technique, the sample is irradiated continuously. The process is performed keeping the e-gun open and shifting the beam energy gradually and automatically. The intensity is measured with an amperemeter. Using this procedure, we obtain the curve of the primary current against the primary energy and the same for the compensatory current. Then, we proceed to calculate the SEY coefficient by using (1).

Here, it is important to stress that when we work with samples with very low conductivity $(\kappa)$, dielectrics for instance, the continuous mode cannot be used due to charge accumulation, and it is necessary that the use of an alternative technique to carry out the measurements.

2) Pulsed Mode: This second technique is based on a different working mode of the e-gun, the pulsed mode. It consists of shutting down the emission of the gun by setting the grid potential at a high level and then shifting it with a function generator. The measured pulses are registered with a transimpedance amplifier and an oscilloscope. Using this technique, we can develop two different analyses.

First of all, we can measure SEY curves on dielectrics, just by sending small charge pulses, in the order of $100 \mathrm{fC}$, and discharging the sample artificially with different methods [16]. The primary energy is shifted manually between pulses.

On the other hand, we can send big charge pulses intentionally, in order to see how the charge affects the secondary emission. In this line, long square primary pulses and also trains of pulses can be used to study the performance of every kind of sample.

\section{FORMULATION}

\section{A. Charging Effects on Pure Insulators}

This section is focused on the physical explanation of the behavior observed in SEY measurements made with a thin Teflon sample. Due to its low conductivity, this material can be assumed to be a pure insulator.

Then, a pure insulator sample can be approximated by a parallel plate capacitor considering that the charge persists during the measurement time. This accumulated charge affects the upcoming incident electrons, in a manner that depends on the charge sign.

First, if the SEY is initially higher than 1, the net electron extraction generates a positive charge on the surface. This leads to a positive potential that reduces the SEE by different mechanisms. The electrostatic potential increases the energy needed to extract electrons from the solid, decreasing electron emission. Furthermore, some part of the emitted electrons return to the sample due to the electrostatic force. These processes lead to a decrease on the outgoing electron number, converging in a steady state where the number of outgoing and the number of incoming electrons become equal; then the effective SEY tends to unity.

On the other hand, when the SEY is initially lower than 1, the net electron injection generates a negative charge in the surface. This produces a negative potential that also change the emission. It decreases the energy needed to extract electrons from the solid, increasing the electron emission. Moreover, 


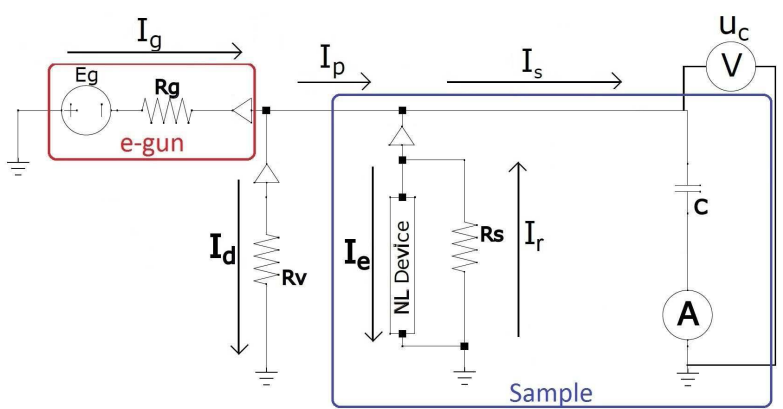

Fig. 2. Equivalent circuit model of the whole experimental setup.

the negative potential reflects some part of the incoming electrons, because of its low energy that is below the first crossover. Both processes lead to a steady state where the effective SEY tends to unity.

After doing these commentaries of the related physical processes, an electric circuit-based model can be used to simplify the entire setup and find out some theoretical solutions for the problem. This kind of circuits has been previously developed in the technical literature as in [10], where a testing circuit for pulsed operation was presented. In this paper, we propose the circuit showed in Fig. 2, which will be explained in the next paragraphs. At this point, we should emphasize that we have solved analytically the circuit of Fig. 2, obtaining theoretical solutions which agree quite well with our experimental results; meanwhile, in [10], the circuit is only used for a detailed description of the experimental setup.

The current sign follows the same criterion fixed in Section II-A. It should be clarified that triangles in Fig. 2 do not mean common diodes, they just indicate some forbidden directions for the current. The amperemeter symbolizes a physical measurement device to evaluate the current $I_{s}$, but the voltimeter does not mean a real device, it is only a symbol to highlight the potential generated on the surface of the sample.

Now, we start describing the circuit; the first branch symbolizes the electron gun, where $I_{g}$ is selected constant for all possible values of primary energy $E_{g}$, so $R_{g}$ will adapt to keep the quotient $E_{g} / R_{g}$ constant. The deflected electron current $I_{d}$ contains some part of the electrons that are emitted by the gun and does not reach the sample, so they are deflected and go to the grounded chamber walls through the vacuum resistance $R_{v}$. Then, the primary current will be $I_{p}=I_{g}-I_{d}$. However, this deflection current only appears for $\sigma<1$ experiments, when the negative potential reflects the electrons.

The component labeled as NL (nonlinear) Device receives the primary current releasing the secondary current $I_{e}$, according to the definition for a noncharged sample $I_{e}=\sigma I_{p}$. The charging effects are taken into account in the response current $I_{r}$; this current will increase or decrease the emission depending on the capacitor potential sign. The sample is initially neutral and $I_{r}(t=0)=0$.

The sample is simplified as a capacitor, and it is charged by the current $I_{S}$. Now, as commented in Section II, $I_{S}$ is positive when the SEY is higher than 1 and then $u_{c}$ will be positive. In this case, $I_{r}=u_{c} / R_{s}>0$ and causes a decay in the emission owing to the processes explained before. Summarizing, $I_{s}=I_{p}-I_{e}+\left|I_{r}\right| \rightarrow 0$ so $\sigma \rightarrow 1$ after enough time. On the other hand, if the SEY is lower than $1, I_{s}$ is negative causing a negative potential. In this case, $I_{r}=u_{c} / R_{s}<0$ and it enforces the emission. To sum up, $I_{s}=I_{p}-I_{e}-\left|I_{r}\right| \rightarrow 0$ and $\sigma \rightarrow 1$ after enough time.

The last step consists of solving the circuit to evaluate the evolution of $I_{s}$ as a function of time. Looking at the previous assumptions, one can establish that

$$
\begin{aligned}
& I_{e}=\sigma I_{p} ; \quad I_{g}=\frac{E_{g}-u_{c}}{R_{g}} \\
& I_{p}=I_{g}-I_{d} ; \quad I_{d}=\frac{u_{c}}{R_{v}} ; \quad \text { only for } \sigma<1 \\
& I_{r}=\frac{u_{c}}{R_{s}} ; \quad I_{s}=-C \frac{d u_{c}}{d t} \\
& I_{s}=I_{p}-I_{e}+I_{r} .
\end{aligned}
$$

Combining these equations into a single differential equation, we obtain

$$
-C \frac{d u_{c}}{d t}=\left[\frac{E_{g}-u_{c}}{R_{g}}-\frac{u_{c}}{R_{v}}\right](1-\sigma)+\frac{u_{c}}{R_{s}}
$$

which can be easily solved, then one finds the solution for the potential

$$
u_{c}(t)=-A+B e^{-t / \tau}
$$

with

$$
\tau= \begin{cases}C\left[\frac{1}{R_{S}}-(1-\sigma)\left(\frac{1}{R_{g}}+\frac{1}{R_{\nu}}\right)\right]^{-1} & \text { for } \sigma<1 \\ C\left[\frac{1}{R_{S}}-(1-\sigma)\left(\frac{1}{R_{g}}\right)\right]^{-1} & \text { for } \sigma>1\end{cases}
$$

where we have to make the limit $R_{v} \rightarrow \infty$ to eliminate the deflection branch for the $\sigma>1$ case. Moreover, A and B are constants. Deriving and applying boundary conditions we finally obtain, the solution we are looking for, i.e.,

$$
I_{S}(t)=\frac{C B}{\tau} e^{-t / \tau}=I_{S}(0) e^{-t / \tau}=I_{p}(1-\sigma) e^{-t / \tau} .
$$

This solution will be compared with the experimental data in Section IV.

\section{B. Sample Discharge Processes}

In this section, we add to the model the possibility of having discharge processes in the sample due to a loss current that may appear in dielectrics with high enough conductivity and semiconductors. Then, the capacitor has a shunted resistor that allows this mechanism. Furthermore, the other components are simplified as shown in Fig. 3.

The new circuit is simpler than the previous one; however, it is evident that, in the approximation of pure insulator $\left(R_{c} \rightarrow \infty\right)$, the solution for $I_{s}(t)$ is formally identical to (6), being $\tau=\mathrm{CR}_{s}$ in this case. This solution is compatible with (5) in the limit case where $R_{g}, R_{v} \gg R_{s}$; this limit will be discussed in Section IV.

In this new case, the measurable current $I_{S}$ is separated into two contributions: $I_{C}$ represents the charging current and $I_{R}$ takes into account the recombination process. A switch is also added to enable the pulsed primary electron emission from the electron gun. Moreover, changing the sign of $V_{0}$, we also change the sign of $I_{s}$. 


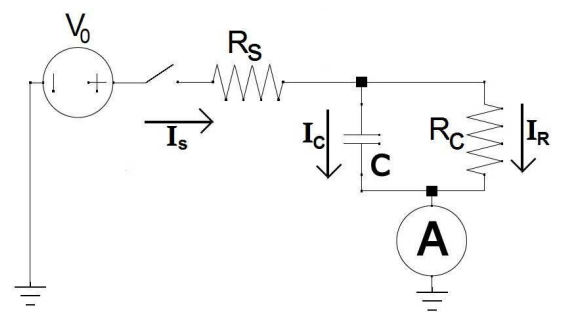

Fig. 3. Simplified circuit used to evaluate the natural charge recombination.

1) Solution in Continuous Mode: The circuit can be solved using Kirchhoff's laws. The current law provides a relation between the intensities of the system

$$
I_{S}=I_{C}+I_{R}
$$

whereas with the voltage law, differential equations are presented in terms of charges

$$
\begin{aligned}
V_{0}-R_{S}\left(\frac{d Q_{C}}{d t}+\frac{d Q_{R}}{d t}\right)-R_{C} \frac{d Q_{R}}{d t} & =0 \\
V_{0}-R_{S}\left(\frac{d Q_{C}}{d t}+\frac{d Q_{R}}{d t}\right)-\frac{Q_{C}}{C} & =0 .
\end{aligned}
$$

Using (7) and (8), an expression for $I_{s}$ is obtained

$$
I_{s}(t)=\underbrace{\frac{V_{0}}{R_{S}} e^{\frac{-t}{\tau_{\mathrm{NN}}}}}_{I_{C}}+\underbrace{\frac{V_{0}}{R_{s}+R_{C}}\left(1-e^{\frac{-t}{\tau_{\mathrm{ON}}}}\right)}_{I_{R}}, \quad \tau_{\mathrm{ON}}=C \frac{R_{s} R_{C}}{R_{s}+R_{C}}
$$

with $\tau_{\mathrm{ON}}$ being the new decay time that incorporates the discharge of the sample.

A representation of the solution found in (9) is presented in Fig. 4(a). The charging current decreases with time as the charge of the capacitor rises until saturation. On the other hand, the recombination current increases reaching a limit value at saturation.

In Fig. 4(b), simulations of $I_{s}$ are presented. Depending on the conductivity, the sample opposes more or less to the recombination. A limit case dealing with metals is presented, where $I_{S}$ keeps constant along the time and the SEY does not change. The opposite happens with dielectric materials where $I_{s}$ tends to 0 , then the SEY tends to 1 in consequence. For dielectrics, (6) is well suited, and the sign shift observed when the SEY is higher or lower than 1 is justified, taking into account that $I_{p}<0$ in our measurements, as explained in Section II-A.

2) Solution in Pulsed Mode: We consider now a pulse set with different sections in ON corresponding to a time $t_{\mathrm{ON}}$ and OFF with $t_{\mathrm{OFF}}$. The ON sections are those where the electron gun is irradiating the sample, and the solution for $I_{s}$ is the same as the one of Sections III-A and III-B1. Nevertheless, OFF sections require a different treatment.

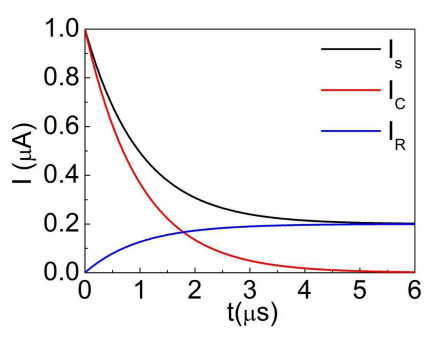

(a)

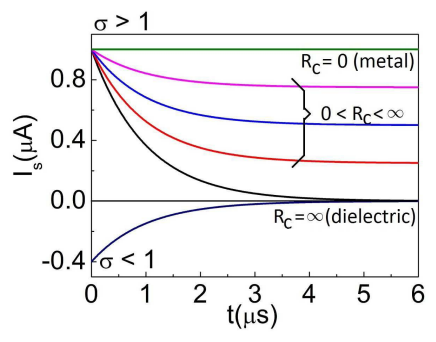

(b)
Fig. 4. (a) Theoretical simulations for $I_{S}$. Different current contributions are presented. (b) Variations with sample's conductivity are shown.

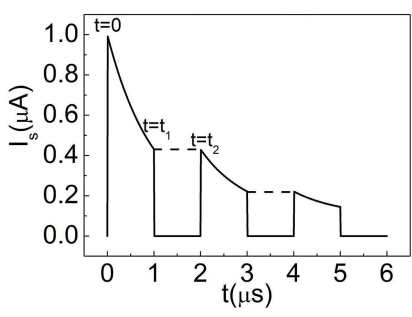

(a)

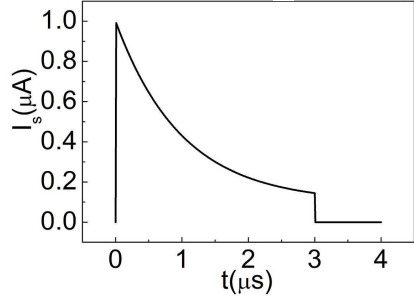

(b)
Fig. 5. Pulsed mode solution for dielectrics. (a) Pulsed mode solution for a pure insulator. (b) Same curve subtracting OFF sections.

In OFF sections, $I_{s}=0$ and therefore $I_{R}=-I_{C}$. If there is no recombination, i.e., for dielectric materials, $\sigma$ maintains constant, since there is no discharge in the OFF sections. This explains that $I_{S}$ is the same at the end of a pulse and at the beginning of the next one, as it is shown in Fig. 5.

Fig. 5 must be translated into a theoretical model, described in terms of the piecewise function defined in (10), as shown at the bottom of this page, where $N \in\left[0, N_{\mathrm{MAX}}\right]$ calls to the $N$ th cell composed by one ON interval plus one OFF interval.

On the other hand, in the case that we analyze metal or semiconductor samples, discharging processes are not negligible. Considering that the circuit is composed by a charged capacitor with an initial charge $Q_{\text {ini }}$, which comes from accumulated charge in previous pulses, and by a resistor $R_{C}$ which links both faces of the capacitor and allows the discharge. The solution of this circuit is

$$
I_{C}^{\mathrm{OFF}}=\frac{-Q_{\mathrm{ini}}}{\tau_{\mathrm{OFF}}} e^{-t / \tau_{\mathrm{OFF}}}, \quad \tau_{\mathrm{OFF}}=R_{C} C .
$$

Using a simple model for the resistor $R_{C}=(1 / \kappa)(d / A)$, and the capacitance $C=\varepsilon(A / d)$, where $A$ is the area of the electron beam on the sample, and $d$ is the thickness of the sample, we notice that $A$ and $d$ vanish and $\tau_{\mathrm{OFF}}$ becomes

$$
\tau_{\mathrm{OFF}}=\frac{\varepsilon}{\kappa}
$$

which is usually called the Maxwell relaxation time.

When the sample is charged, the effective SEY changes. Therefore, if the sample becomes neutral, the SEY will recover its initial value $\sigma$. Now, the charging current is known in both

$$
I_{s}(t)= \begin{cases}I_{s}\left(t-N t_{\mathrm{OFF}}\right) & \text { if } t \in\left[N t_{\mathrm{ON}}+N t_{\mathrm{OFF}},(N+1) t_{\mathrm{ON}}+N t_{\mathrm{OFF}}\right] \\ 0 & \text { if } t \in\left[(N+1) t_{\mathrm{ON}}+N t_{\mathrm{OFF}},(N+1) t_{\mathrm{ON}}+(N+1) t_{\mathrm{OFF}}\right]\end{cases}
$$




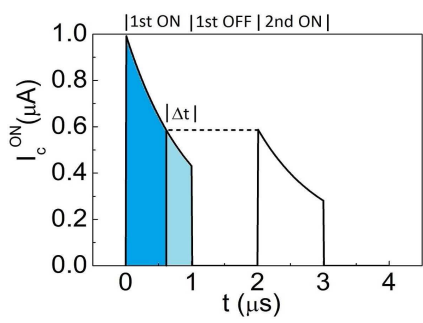

(a)

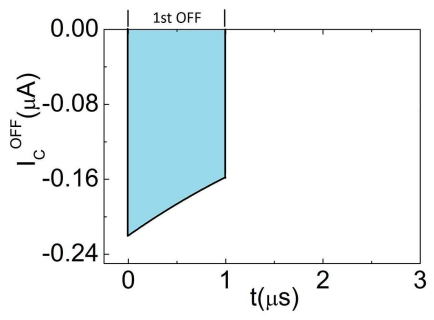

(b)
Fig. 6. Representation of (a) ON charging current and (b) OFF discharging current. The remarked areas symbolize the charge accumulated (both blue) and recombined (light blue).

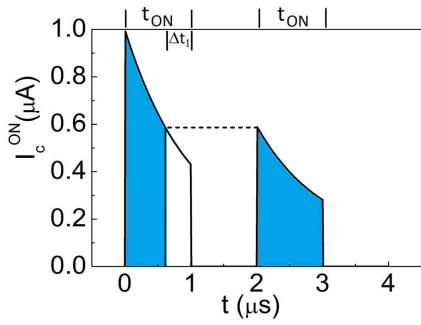

(a)

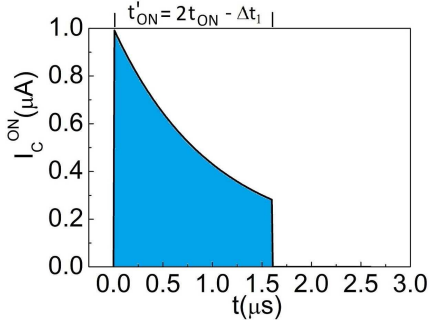

(b)
Fig. 7. (a) Accumulated charge after two pulses taking into account the recombination. (b) Equivalent pulse.

the cases: when the electron gun irradiates the sample (ON) and when it discharges by itself (OFF). The charge state of the sample can be evaluated by integrating the current in the corresponding intervals.

As showed in Fig. 6(a), the blue areas symbolize the accumulated charge in the first ON, whereas the light blue area in both curves explains the recombined charge in the first OFF. Therefore, an increase in $I_{S}$ is expected in the following pulse.

The discharging process produces a time-shift $\Delta t$. This time can be known evaluating the integrals analytically obtaining

$$
\Delta t=\tau_{\mathrm{ON}} \ln \left[1+\left(e^{\frac{t_{\mathrm{ON}}}{\tau_{\mathrm{ON}}}}-1\right)\left(1-e^{\frac{-t_{\mathrm{OFF}}}{\tau_{\mathrm{OFF}}}}\right)\right] .
$$

However, (13) is only valid for the first OFF section. It may be observed that in the limit of complete recombination $t_{\mathrm{OFF}} \gg$ $\tau_{\mathrm{OFF}}$ and $\Delta t \rightarrow t_{\mathrm{ON}}$. This means that $\sigma$ recovers its initial value. For successive pulses, the sample accumulates more charge as shown in Fig. 7. The final accumulated charge is equivalent to the one after sending a unique pulse of length $t_{\mathrm{ON}}^{\prime}=2 t_{\mathrm{ON}}-\Delta t_{1}$, where $\Delta t_{1}$ means the time-shift due to recombination for the first OFF interval. In the general development that follows, we will use $\Delta t_{i}$ for the $i$ th OFF interval.

Taking this into account, the model can be generalized for whatever pulse as follows:

$$
\begin{aligned}
t_{\mathrm{ON}}^{N} & =N t_{\mathrm{ON}}-\sum_{i=1}^{N-1} \Delta t_{i} \\
\Delta t_{N} & =\tau_{\mathrm{ON}} \ln \left[1+\left(e^{\frac{t_{\mathrm{N}}^{N}}{\tau_{\mathrm{ON}}}}-1\right)\left(1-e^{\frac{-t_{\mathrm{OFF}}}{\tau_{\mathrm{OFF}}}}\right)\right] \\
I_{s}^{\mathrm{pulse} N+1}(t) & =I_{s}\left(t-\sum_{i=1}^{N} \Delta t_{i}\right) .
\end{aligned}
$$

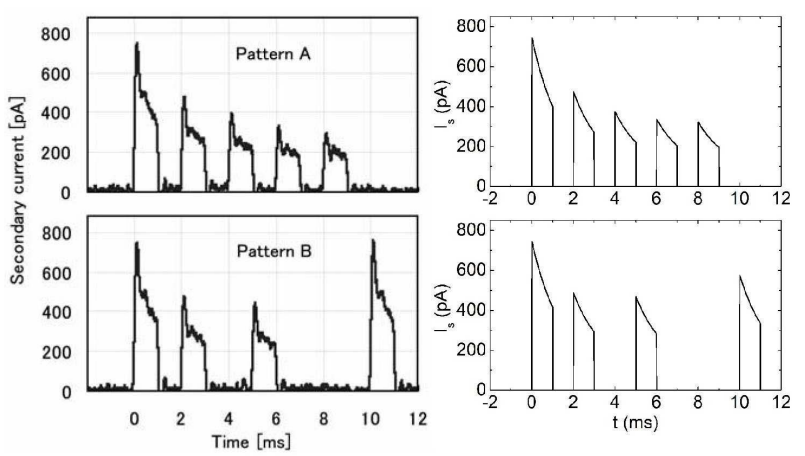

Fig. 8. Theoretical simulations for the measurements presented in [17]. Left column: experimental measurements. Right column: simulations obtained with the proposed model.

To clarify the previous equations, it is important to stress that the first pulse corresponds to $N=0$ and does not require the use of (14), since $\Delta t=0$. The second pulse is the first where the effect of the recombination appears, it is identified with $N=1$ and (13) must be used. For the third and successive pulses, the general expressions of (14) are suitable from $N=2$.

This model is compared in Fig. 8 with experimental measurements presented in [17], finding good agreement.

\section{RESUlts}

Once the experimental setup and the proposed equivalent model have been presented, we introduce in this section the evolution with time of the secondary emission of the samples as well as its dependence with the primary energy.

\section{A. Evolution in Time of the Secondary Emission}

1) Teflon Irradiated With DC: We start presenting the results obtained for the Teflon sample. Fig. 9 shows the evolution with time of $I_{s}$ for a primary square pulse of $60-\mu \mathrm{s}$ length. This pulse is long enough to observe the saturation of the sample, so this situation is equivalent to dc irradiation. Moreover, in Fig. 9(a), we sent electrons with $E_{p}=272 \mathrm{eV}$ (300 eV in the e-gun applying the correction of the bias voltage) and this primary energy leads to a $\sigma>1$ situation. On the other hand, in Fig. 9(b), $E_{p}=12 \mathrm{eV}$ and this leads to a $\sigma<1$ situation with the corresponding change in the current sign.

In Fig. 9(a) and (b), $I_{s} \rightarrow 0$ and, in consequence, $\sigma \rightarrow 1$. Furthermore, an exponential function fits the transient very good. All these facts are in agreement with the theoretical predictions presented in Section III-A.

The higher noise-to-signal ratio observed in Fig. 9(b) is justified, since with the same noise contributions, we measure a lower signal amplitude. This occurs because we are near the first crossover, so $I_{p}$ and $I_{e}$ are close in magnitude and $I_{s}$ finds here its minimum value.

Now, we detail the fitting values for the decay time: $\tau_{272 \mathrm{eV}}=3.75 \mu \mathrm{s}$ and $\tau_{12 \mathrm{eV}}=3.46 \mu \mathrm{s}$. These values are very close and verify the limit case $\tau \approx \mathrm{CR}_{s}$, applied to justify the simplified circuit of Fig. 3. We could never find that 


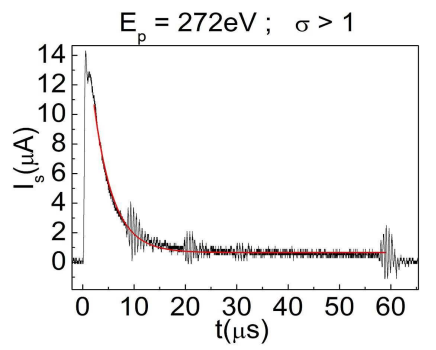

(a) (b)

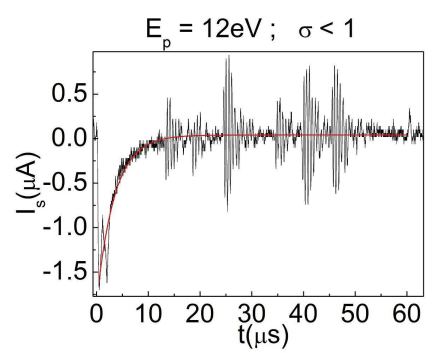

Fig. 9. Experimental curves and fitting (red line) of $I_{S}$ obtained for the Teflon sample. Different primary energies are used leading (a) $\sigma>1$ and (b) $\sigma<1$.

TABLE I

Conductivities of THE SAMPLES

\begin{tabular}{|c|c|}
\hline Material & $\kappa(\mathrm{S} / \mathrm{m})$ \\
\hline $\mathrm{Pt}[19]$ & $9.43 \cdot 10^{6}$ \\
\hline $\mathrm{Si}[20]$ & $10^{2}$ \\
\hline GaAs [21] & $10^{-10}$ \\
\hline Teflon [18] & $10^{-16}-10^{-22}$ \\
\hline
\end{tabular}

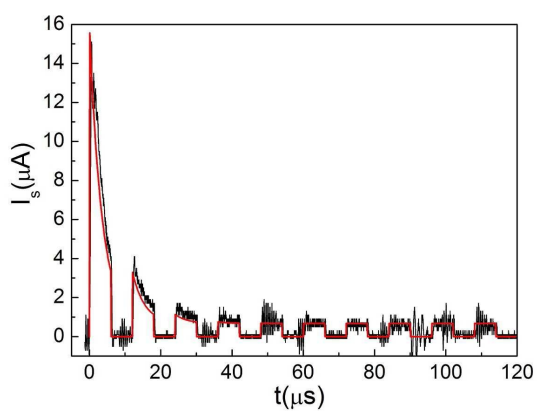

Fig. 10. Experimental curves and theoretical simulation (red line) of $I_{S}$ obtained for the Teflon sample irradiated with a train of pulses.

$\tau_{272 \mathrm{eV}} \approx \tau_{12 \mathrm{eV}}$ using (5), since there is a different expression for each case; unless $R_{g}$ and $R_{v}$ are higher than $R_{s}$, the term that depends on $\sigma$ vanishes.

2) Teflon Irradiated With a Pulse Train: Now, 10 pulses of $6-\mu \mathrm{s}$ width and $6 \mu$ s between them are sent to the sample. The results are presented in Fig. 10 together with the corresponding theoretical simulation (red line), obtained using the model of Section III-B2. In this simulation, we have supposed that no recombination occurs in OFF intervals, and the sample remains in the same state. The good agreement between theory and experiment indicates that the natural recombination of the Teflon sample cannot be observed in this range of waiting times.

This is understood taking into account the properties of the Teflon material obtained from [18]: $\varepsilon=2.1$ and $\kappa$ according to Table I. Making the calculation of $\tau_{\mathrm{OFF}}=(\varepsilon / \kappa)$, the Maxwell relaxation time is obtained among 2 days and 1000 years.

3) All Samples Comparison, Irradiating With DC: With the objective of finding samples with natural recombination, $\mathrm{GaS}$ and $\mathrm{Si}$ samples are studied, since they are semiconductors and their conductivity is higher than that of the Teflon sample. However, their conductivity is lower compared with

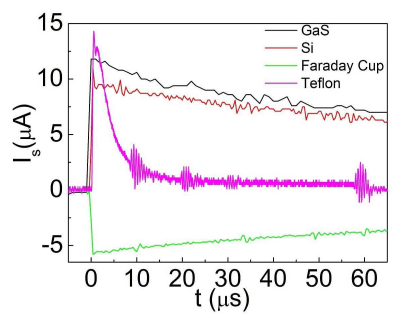

(a)

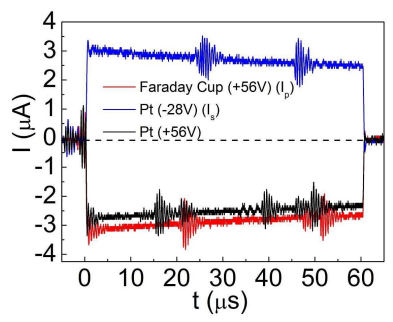

(b)
Fig. 11. (a) Comparison of the secondary currents measured in $\mathrm{Si}, \mathrm{GaS}$, and Teflon. (b) Calibration of the experiment using a metal sample (Pt).

TABLE II

MAin Parameters of the SEY CURVES OF FIG. 12

\begin{tabular}{|c|c|c|c|}
\hline & $E_{1}(\mathrm{eV})$ & $E_{M}(\mathrm{eV})$ & $\sigma_{M}$ \\
\hline Pt (continuous) & $123.6 \pm 0.8$ & $559.7 \pm 0.8$ & $1.77 \pm 0.05$ \\
\hline Pt (pulsed) & $124.7 \pm 0.8$ & $559.1 \pm 0.8$ & $1.78 \pm 0.08$ \\
\hline Si (continuous) & $23.1 \pm 0.8$ & $211.4 \pm 0.8$ & $1.91 \pm 0.05$ \\
\hline Si (pulsed) & $23.6 \pm 0.8$ & $228.1 \pm 0.8$ & $1.91 \pm 0.06$ \\
\hline GaS (continuous) & $32.7 \pm 0.8$ & $306.1 \pm 0.8$ & $2.24 \pm 0.05$ \\
\hline GaS (pulsed) & $25.9 \pm 0.8$ & $308.4 \pm 0.8$ & $2.37 \pm 0.06$ \\
\hline Teflon (pulsed) & $16.2 \pm 0.8$ & $298.6 \pm 0.8$ & $2.73 \pm 0.05$ \\
\hline
\end{tabular}

metals $(\mathrm{Pt})$, and then some charging effects might appear. Results are shown in Fig. 11(a), where no charging effects are visible in $\mathrm{Si}$ and $\mathrm{GaS}$, since it can be seen that $I_{S}$ is proportional to $I_{p}$, the primary pulse measured with the Faraday cup. Moreover, the decay observed in the primary pulse is induced by the electron gun; a capacitive box allows to send short pulses but does not give the possibility to send long square pulses, because it accumulates charge and the electron emission decays. Teflon data have been included in Fig. 11(a) to highlight the faster decay presented due to charging effects in the sample.

In Fig. 11(b), a calibration with $\mathrm{Pt}$ is presented. $I_{p}$ taken with the positive-biased Farday cup corresponds to the red line, whereas the black line is a test current measured in a positive-biased Pt sample. This current is similar to $I_{p}$, and it means that the electron beam is well focused on the sample; the small discrepancy between these two biased situations is caused by some backscattered electrons that escape and do not contribute to the signal. Finally, the blue one is $I_{s}$ measured for the Pt sample, and it is proportional to $I_{p}$, because no charging effects appear in metals.

\section{B. SEY Against Primary Energy Measurements}

To continue with the analysis, $\sigma$ versus $E_{p}$ curves for platinum, silicon, gallium sulfide, and Teflon are made, comparing both continuous and pulsed mode. The results are presented in Fig. 12. To complement the information of this figure, the main parameters of these $\sigma$ curves are presented in Table II. These parameters are the first crossover $E_{1}$, the maximum value of the yield $\sigma_{M}$, and the energy at which this value is reached $E_{M}$. All these parameters were obtained fitting polynomial curves to the scatter plots.

According to Fig. 12, in $\mathrm{Pt}$ and $\mathrm{Si}$, it can be seen that there is no discrepancy between both the modes, whereas for the GaS curve, the continuous mode curve (blue line) is clearly below 

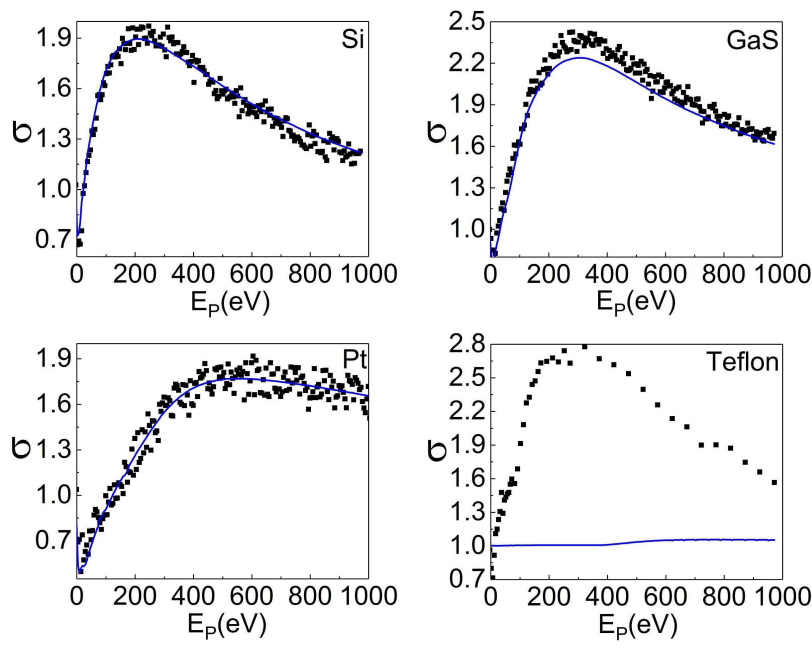

Fig. 12. SEY curves of $\mathrm{Si}, \mathrm{GaS}, \mathrm{Pt}$, and Teflon in a continuous mode (blue curve) and a pulsed mode (black dots).

the pulsed mode black dots. Moreover, for Teflon results, the discrepancy is even higher. Indeed, we observe that $\sigma \approx 1$, as discussed in theory and measured in the Teflon transients.

If we now compare the conductivities of all samples, according to Table I, it is evident that the natural discharge needs more time when the conductivity becomes lower, because, as seen in (12), $\tau_{\mathrm{OFF}} \propto \kappa^{-1}$. Therefore, the differences in the pulsed and continuous curves can be explained taking into account that, in the pulsed mode, the sample is able to recombine itself. Instead, the continuous mode does not let the sample to recombine. It is also important to point out that in Teflon, the recombination is not produced naturally but artificially.

\section{Conclusion}

In this paper, a theoretical analysis of the processes of surface charging on dielectric materials has been developed, as well as the consequences that they generate in SEY measurements. Inspired by experimental measurements and modeling the setup with an equivalent circuit, it has been possible to study all these effects.

From our results, one can understand why the SEY tends to 1 with an exponential transient in insulators, independent of whether the starting value is higher or lower than 1 . On the other hand, in metals and semiconductors, this variation is not as sharp or directly negligible.

Furthermore, the pulsed mode problem has helped to quantify the charging state of the sample after each pulse. It has also aided to evaluate the time expected by the sample to be discharged naturally, since now it is clear that this is near the order of the Maxwell relaxation time and it depends on the material properties.

All these analyses are also useful to clarify the working ranges for pulsed and continuous mode, since it was observed that with Pt and Si samples, the continuous mode is suitable, whereas for the $\mathrm{GaS}$ and Teflon samples, the pulsed mode is more appropriate.

\section{ACKNOWLEDGMENT}

The authors would like to thank the European High Power Space Materials Laboratory for its contribution-a laboratory funded by the European Regional Development Fund-a way of making Europe. Many thanks to the University of Valencia (Spain) for supporting this research activity with the internal program "Assistance for temporary stays of invited researchers within the framework of the Subprogramme Attraction of Talent 2015".

\section{REFERENCES}

[1] J. R. M. Vaughan, "Multipactor," IEEE Trans. Electron Devices, vol. ED-35, no. 7, pp. 1172-1180, Jul. 1988.

[2] L. Wu and L. K. Ang, "Multipactor discharge in a dielectric-loaded accelerating structure," Phys. Plasmas, vol. 14, no. 1, p. 013105, 2007.

[3] C. Vicente et al., "Multipactor analysis in microwave components for high-power satellite applications," in Proc. Conf. Rec. 27th Int. Power Modulator Symp., May 2006, pp. 385-388.

[4] E. Sorolla, A. Sounas, and M. Mattes, "Space charge effects for multipactor in coaxial lines," Phys. Plasmas, vol. 22, no. 3, p. 033512, 2015 .

[5] A. M. Pérez et al., "Multipactor analysis in coaxial waveguides for satellite applications using frequency-domain methods," in IEEE MTT-S Int. Microw. Symp. Dig., Jun. 2006, pp. 1045-1048.

[6] C. Bourat and J.-M. Joly, "On multipactor effect in a $600 \mathrm{MHz} \mathrm{RF}$ cavity used in electron linear accelerator," IEEE Trans. Electr. Insul., vol. 24, no. 6, pp. 1045-1048, Dec. 1989.

[7] R. J. Burton, M. S. de Jong, and L. W. Funk, "Vacuum and multipactor performance of the hadron electron ring accelerator $52 \mathrm{MHz}$ cavities," J. Vac. Sci. Technol. A, Vac., Surf., Films, vol. 9, no. 3, p. 2081, 1991.

[8] J. de Lara et al., "Multipactor prediction for on-board spacecraft RF equipment with the MEST software tool," IEEE Trans. Plasma Sci., vol. 34, no. 2, pp. 476-484, Apr. 2006.

[9] H. Hintenberger, "Über Sekundärelektronenemission und Aufladungserscheinungen an Isolatoren," Zeitschrift Phys., vol. 114, pp. 98-109, Jun. 1939.

[10] J. B. Johnson, "Secondary electron emission from targets of bariumstrontium oxide," Phys. Rev., vol. 73, no. 9, p. 1058, May 1948.

[11] I. M. Bronshtein and B. S. Fraiman, Vtorichnaya Elektronnaya Emissiya (Secondary Electron Emission). Moscow, Russia: Nauka, 1969.

[12] E. Bronchalo et al., "Secondary electron emission of Pt: Experimental study and comparison with models in the multipactor energy range," IEEE Trans. Electron Devices, vol. 63, no. 8, pp. 3270-3277, Aug. 2016.

[13] J. Cazaux, "A new model of dependence of secondary electron emission yield on primary electron energy for application to polymers," J. Phys. D: Appl. Phys., vol. 38, no. 14, pp. 2433-2441, 2005.

[14] N. Balcon, D. Payan, M. Belhaj, T. Tondu, and V. Inguimbert, "Secondary electron emission on space materials: Evaluation of the total secondary electron yield from surface potential measurements," IEEE Trans. Plasma Sci., vol. 40, no. 2, pp. 282-290, Feb. 2012.

[15] Val Space Consortium. Accessed: Feb. 28, 2018. [Online]. Available: http://www.val-space.com/

[16] M. Belhaj, T. Tondu, and V. Inguimbert, "Experimental investigation of the effect of the internal space charge accumulation on the electron emission yield of insulators submitted to e-irradiation: Application to polycrystalline MgO," J. Phys. D: Appl. Phys., vol. 42, no. 14, p. 145306, 2009.

[17] S. Suharyanto, Y. Yamano, S. Kobayashi, S. Michizono, and Y. Saito, "Secondary electron emission and surface charging evaluation of alumina ceramics and sapphire," IEEE Trans. Dielectr. Electr. Insul., vol. 13, no. 1, pp. 72-78, Feb. 2006.

[18] Teflon Ptfe Fluoropolymer Resin: Properties Handbook. DuPont Fluoroproducts, 1996. [Online]. Available: http://www.rjchase.com/ ptfe_handbook.pdf

[19] R. A. Serway, Principles of Physics, 2nd ed. London, U.K.: Saunders, 1998.

[20] Topsil Global Wafers A/S. Accessed: Feb. 28, 2018. [Online]. Available: http://www.topsil.com/

[21] O. Madelung and U. Rössler, Non-Tetrahedrally Bonded Elements and Binary Compounds I (Landolt-Börnstein), vol. 41C. Berlin, Germany: Springer, 1998. 